\title{
Elasticity generates indissoluble biomolecular condensates
}

\author{
Lingyu Meng ${ }^{1}$ and Jie Lin ${ }^{1,2}$ \\ ${ }^{1}$ Peking-Tsinghua Center for Life Sciences, Peking University, Beijing, China \\ ${ }^{2}$ Center for Quantitative Biology, Peking University, Beijing, China
}

(Dated: February 10, 2022)

\begin{abstract}
While biomolecular condensates are often liquid-like, many experiments found that condensates also exhibit solid-like behaviors, which may make them irreversible and indissoluble. Despite the critical biological significance of indissoluble condensates to cellular fitness and diseases, the physical mechanisms underlying the stabilities of solid-like condensates are still unclear. In this work, we study the effects of elasticity on the dissolution of biomolecular condensates. We demonstrate that the bulk stress inside condensates may prevent the condensates from dissolution and obtain the new equilibrium conditions of elastic condensate: the osmotic pressure minus the bulk stress is uniform inside and outside condensates. To verify our theories, we simulate the two-fluid model in which the slow component corresponding to the polymer network generates elastic stress. Our theoretical predictions are nicely confirmed and independent of microscopic details. Moreover, we obtain a phase diagram on the stability of elastic condensates and identify a minimum bulk modulus for the condensates to be indissoluble, both numerically and theoretically. Our results may have implications in developing drugs targeting irreversible condensates.
\end{abstract}

19 20 ous organisms, usually composed of proteins and RN ${ }_{21}[1-9]$. They often have crucial biological functions $22[10,11]$, such as adaptive responses to stresses, acceler23 ating biochemical reactions, and sequestering molecules 24 from reactions. Therefore, the accurate regulation of 25 biomolecular condensates' formation and dissolution is ${ }_{26}$ critical. Meanwhile, experiments have also found that 27 biomolecular condensates are viscoelastic rather than ${ }_{28}$ completely viscous [12]: they are solid-like on a short 29 time scale and liquid-like on a long time scale. More 30 interestingly, they exhibit aging behaviors, and the vis31 coelastic relaxation time, which separates solid and liquid 32 behaviors, increases over time [13]. Indeed, aged conden33 sates may become indissoluble or infusible in conditions 34 where newly formed condensates can easily dissolve or ${ }_{35}$ fuse $[3,4,7,9,14-18]$. Moreover, indissoluble conden36 sates may affect cellular fitness, e.g., failure to dissolve 37 condensates during mitosis leads to aberrant condensates 38 that cause the cell-cycle arrest and ultimately cell death 39 [19]. In general, indissoluble condensates are believed to 40 lead to aging at the cellular or organismic level and are 41 related to multiple diseases [15, 20-22].

42 Theoretical studies on the formation and dissolution 43 of biomolecular condensates have so far been limited to 44 fluid models, in which the elastic nature of condensates 45 are usually neglected [23]. Meanwhile, numerous exper46 iments have demonstrated the importance of solid-like 47 nature on the dissolution of condensates [13, 22], and 48 our theoretical understanding of this problem is still in 49 its infancy. In this work, we seek to fill this gap and in50 vestigate the effects of elasticity on the dissolution and 51 stability of biomolecular condensates, combining both 52 analytical theories and computer simulations. In the 53 following, we first introduce our theoretical frameworks ${ }_{54}$ focusing on elastic condensates that are subject to an
${ }_{55}$ abrupt parameter change. Without elasticity, they are 56 supposed to dissolve. We then derive the equilibrium 57 conditions for elastic condensates and find that the bulk 58 stress plays an essential role in preventing the dissolu59 tion. A finite bulk modulus may render the condensate 60 irreversible and indissoluble. To test our theoretical pre${ }_{61}$ dictions, we simulate the two-fluid model [24, 25] beyond 62 the traditional fluid model of phase separation dynamics ${ }_{63}$ (Model H) [23, 26], by including elastic stress generated 64 by the polymer network. Our theories are nicely con${ }_{65}$ firmed and valid independent of the microscopic details, 66 such as the free energy form. Furthermore, we provide a 67 phase diagram of condensate stability and demonstrate 68 a minimum bulk modulus for the condensate to be indis69 soluble. Finally, we discuss the biological implications of 70 our work and propose future directions to explore.

\section{$71 \quad$ Equilibrium conditions of elastic condensates}

72 Biomolecular condensates usually have well-defined 73 viscoelastic relaxation times, below which the conden74 sates behave as elastic materials $[12,13]$. In this work, we 75 simplify the problem by considering an aged condensate 76 with its viscoelastic relaxation time much longer than 77 the time scales of biological interests, e.g., the duration 78 of cell-cycle phases. Therefore, its viscoelastic relaxation 79 time can be taken as infinite, which is the main focus 80 of this work (Figure 1). We introduce an abrupt change ${ }_{81}$ to the attractive interaction between the monomers of 82 biomolecules, either due to environmental change, e.g., 83 an increase in temperature, or active cellular regulation, ${ }_{84}$ e.g., post-translational phosphorylation. In the absence 85 of elasticity, the condensate will dissolve. However, as we 86 show in the following, the elastic force may prevent the 87 dissolution. 

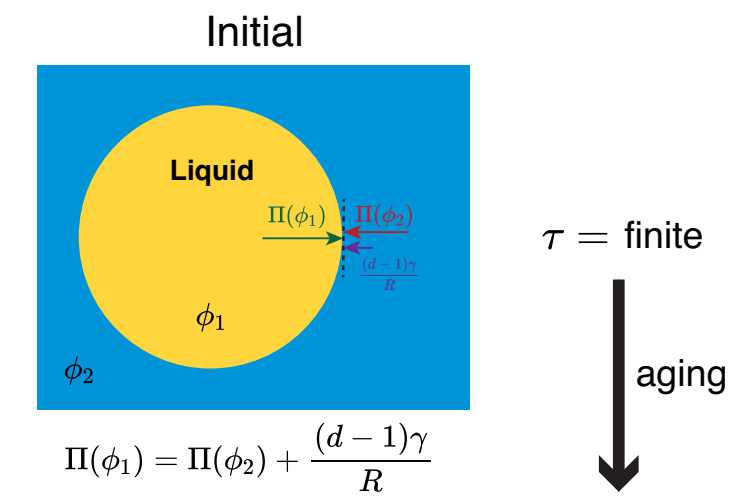

Final

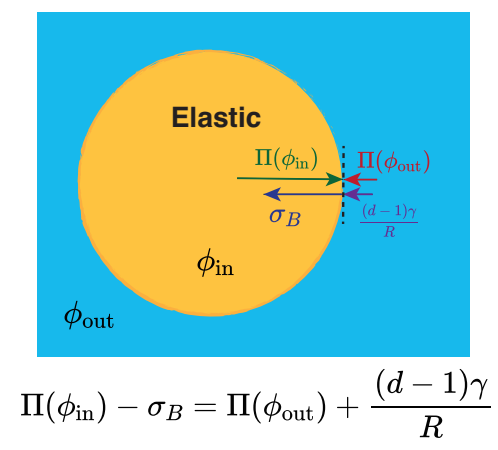

$\tau=\infty$

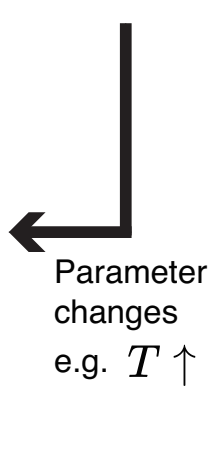

FIG. 1. Equilibrium conditions of elastic condensates. Liquidlike condensates are initially formed from liquid-liquid phase separation, which then become aged and solid-like. An abrupt change in the effective attractive interaction between biomolecular monomers is introduced, e.g., through an increase in temperature or post-translational modification. The bulk stress $\sigma_{B}$ in the condensate is involved in the new equilibrium condition to prevent dissolution.
102 which is responsible for the bulk stress. $G_{B}$ is the bulk 103 modulus. In writing the above two equations, we assume 104 that $\phi$ and $\sigma_{B}$ are uniform inside the condensate, which 105 we confirm numerically later. Combining Eqs. $(2,3)$, we 106 obtain

$$
\sigma_{B}=G_{B} \ln \left(\frac{\phi_{1}}{\phi_{\text {in }}}\right)
$$

${ }_{107}$ where $\phi_{1}$ and $\phi_{\text {in }}$ are respectively the densities of the 108 condensate before and after the condition changes.

${ }_{109}$ We note that to uniquely determine the density inside 110 and outside the condensate, $\phi_{\text {in }}$ and $\phi_{\text {out }}$, we still need 111 one more equation. For liquid condensates, it is a uniform 112 chemical potential. However, in our case, the conden113 sate is solid; therefore, the exchange of molecules is sup114 pressed $[3,8,22]$. Instead, we propose that condensate 115 size does not change upon the weakening of attractive in116 teraction between biomolecules' monomers, namely, $R=$ ${ }_{117} R_{0}$, where $R$ and $R_{0}$ are respectively the radii of elastic 118 condensate before and after the condition changes. We 119 confirm this assumption numerically later. Given $R$ and ${ }_{120} \phi_{\text {in }}, \phi_{\text {out }}$ can be calculated using the conservation of total 121 molecular number: $V \phi_{0}=\sum_{i} V_{i} \phi_{\text {in }, i}+\left(V-\sum_{i} V_{i}\right) \phi_{\text {out }}$. ${ }_{122}$ Here $V$ is the total volume, $V_{i}$ is the volume of conden123 sate $i$, and the summation is over all condensates. $\phi_{0}$ is ${ }_{124}$ the average density over the total volume. Finally, we 125 remark that in our case, the bulk stress inside the con126 densate stabilizes the condensate, in contrast to the bulk 127 stress outside a condensate, e.g., due to the surrounding 128 polymer network that suppresses the formation of con129 densates [27-29].

${ }_{88}$ In liquid-liquid phase separation, a stable condensate 89 requires the Gibbs-Thomson relation of osmotic pressure: ${ }_{90} \Pi_{\text {in }}=\Pi_{\text {out }}+(d-1) \gamma / R$. Here, $\Pi_{\text {in (out) }}$ is the osmotic ${ }_{91}$ pressure inside (outside) the condensate, $\gamma$ is the surface ${ }_{92}$ tension constant, $d$ is the spatial dimension and $R$ is the ${ }_{93}$ condensate radius. In this work, we propose the following 94 equilibrium condition for elastic condensates (see detailed 95 derivations in Methods),

$$
\Pi_{\mathrm{in}}-\sigma_{B}=\Pi_{\mathrm{out}}+\frac{(d-1) \gamma}{R}
$$

${ }_{96}$ Here $\sigma_{B}$ is the bulk stress inside the condensate (Figure ${ }_{97} 1$ ). As we show later, the inclusion of bulk stress com98 pensates the imbalance of osmotic pressures. To find the 99 expression of $\sigma_{B}$, we use the constitutive equation of the 100 bulk stress and the continuity equation of density $(\phi)$

$$
\begin{aligned}
\frac{\partial \sigma_{B}}{\partial t} & =G_{B} \nabla \cdot \mathbf{v}_{\mathbf{p}} \\
\frac{\partial \phi}{\partial t} & =-\phi \nabla \cdot \mathbf{v}_{\mathbf{p}} .
\end{aligned}
$$

${ }_{140}$ Here, $\zeta$ is the friction constant between polymer and sol141 vent, and $\eta$ is the viscosity. The pressure $p$ is determined 142 by the incompressible condition: $\nabla \cdot \mathbf{v}=0$. The stress (3) 143 tensor $\sigma=\sigma_{S}+\sigma_{B} \mathbf{I}$ where $\sigma_{B}$ is the bulk stress and ${ }_{144} \sigma_{S}$ is the shear stress tensor. They follow the Maxwell 101 Here $\mathbf{v}_{\mathbf{p}}$ is the velocity field of the polymer, e.g., proteins, ${ }_{145}$ fluid dynamics with the bulk and shear modulus $G_{B}$ and 
a

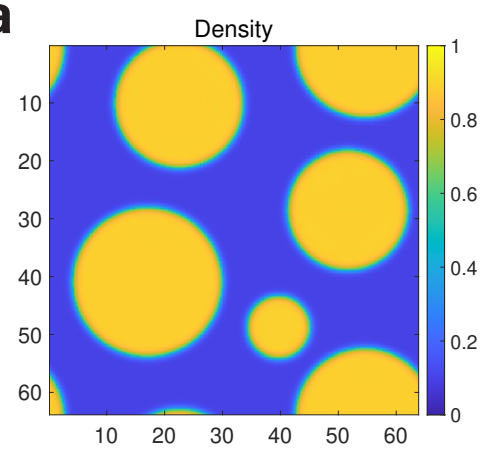

b

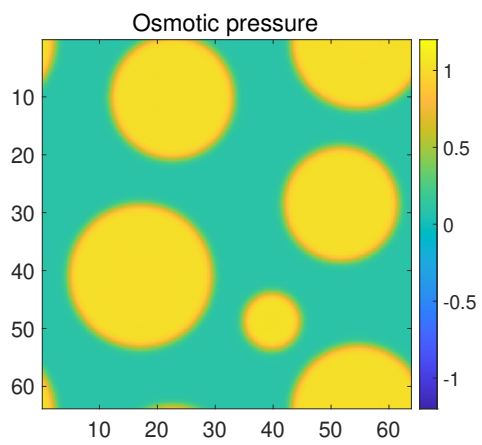

C

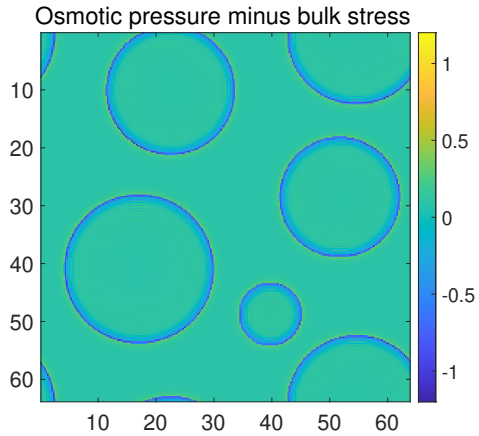

FIG. 2. Simulations of multiple coexisting condensates. (a) The density field $\phi$ after decreasing $\chi$ from 3.0 to 1.5 . (b) The osmotic pressure $\Pi$ from the same simulation of (a). (c) $\Pi-\sigma_{B}$ from the same simulation of (a). In this figure, we take $\phi_{0}=0.45, G_{B}=20, G_{S}=20$, and $\phi_{c}=0.5$.

a

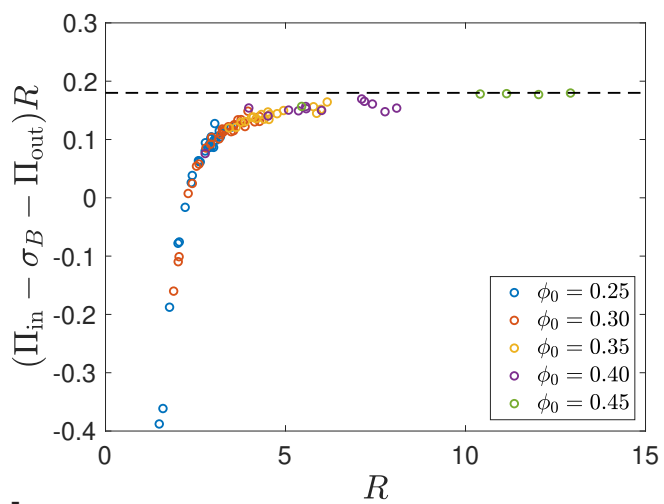

b

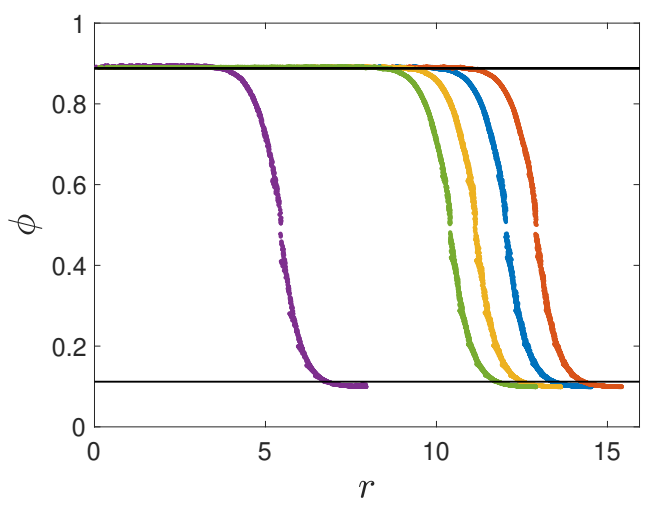

${ }_{147}$ sates, we take the relaxation time of bulk stress to be 148 diverging at a critical density $\phi_{c}$ such that

$$
\tau_{B}^{-1}(\phi)=\left(\phi_{c}-\phi\right) \Theta\left(\phi_{c}-\phi\right),
$$

149 where $\Theta(x)$ is the Heaviside function. We remark that if 150 we take the stress tensor to be zero in Eqs. $(5,6)$, they 151 are reduced to the classical Model H [26]. The osmotic 152 stress tensor is determined by the polymer free energy ${ }_{153} f(\phi), \nabla \cdot \boldsymbol{\Pi}=\phi \nabla f^{\prime}(\phi)$ where $f(\phi)=f_{0}(\phi)+\frac{C}{2}(\nabla \phi)^{2}$ and ${ }_{154} C$ is a constant. If not mentioned explicitly, we use the ${ }_{155}$ Flory-Huggins free energy: $f_{0}=\phi \ln (\phi)+(1-\phi) \ln (1-$ $\left.{ }_{156} \phi\right)+\chi \phi(1-\phi)$. The condition of stable liquid condensate ${ }_{157}$ is that the control parameter $\chi>2$. In this work, we use 158 the osmotic pressure $\Pi$ to represent the scalar osmotic 159 stress computed from $f_{0}, \Pi=\phi f_{0}^{\prime}-f_{0}$.

${ }_{160}$ We simulate multiple coexisting elastic condensates by ${ }_{161}$ changing $\chi$ from $\chi_{i}=3$ to $\chi_{f}=1.5$. According to our 162 theories, they can be simultaneously stable if each has its 163 osmotic pressure difference balanced by the bulk stress. ${ }_{164}$ The density field is indeed uniform inside condensates as 165 assumed (Figure 2a). We also confirm our assumptions 166 of uniform bulk stress and constant radii (Figure S1). ${ }_{167}$ Example of simulations are shown in Movie S1. We find 168 that the osmotic pressure is significantly different across 169 the boundaries of condensates (Figure 2b). For liquid 170 condensates, they will quickly dissolve due to the large 171 pressure difference. In contrast, the bulk stress balances 172 the osmotic pressure difference for elastic condensates. ${ }_{173}$ Indeed, we find that the $\Pi-\sigma_{B}$ field is uniform across 174 the boundaries (Figure 2c). sion constant $\gamma=\left(\Pi_{\mathrm{in}}-\sigma_{B}-\Pi_{\mathrm{out}}\right) R$ approaches an asymptotic value in the large radius limit. (b) A comparison of the theoretical predictions of $\phi_{\text {in }}$ and $\phi_{\text {out }}$ (black lines) and the simulations in Figure 2(a). Each colored curve represents one condensate and $r$ is the distance from the condensate center. In both (a) and (b), $G_{B}=20, G_{S}=20, \phi_{c}=0.5, \chi_{i}=3.0$, $\chi_{f}=1.5$. In (b), $\phi_{0}=0.45$.

${ }_{146} G_{S}$ (Methods). In particular, to simulate elastic conden- ${ }_{183}$ our predictions of the density $\phi_{\text {in }}$ and $\phi_{\text {out }}$ assuming $\gamma=$ 
a

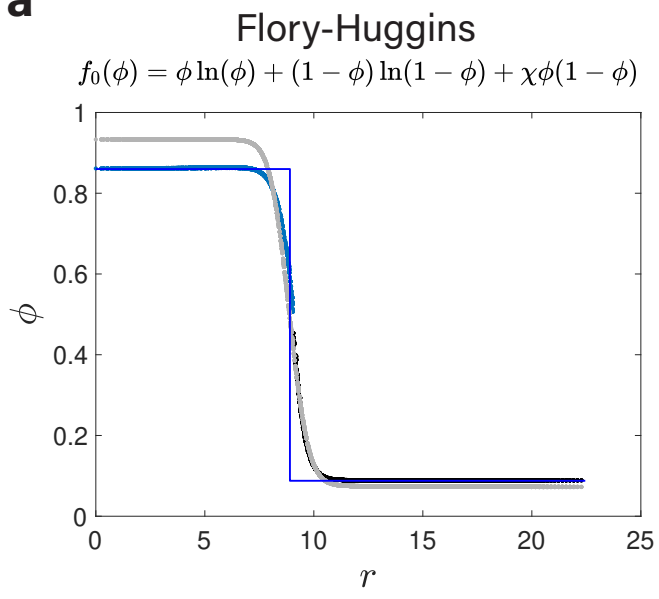

$\chi_{i}=3 \longrightarrow \chi_{f}=1.5$

b

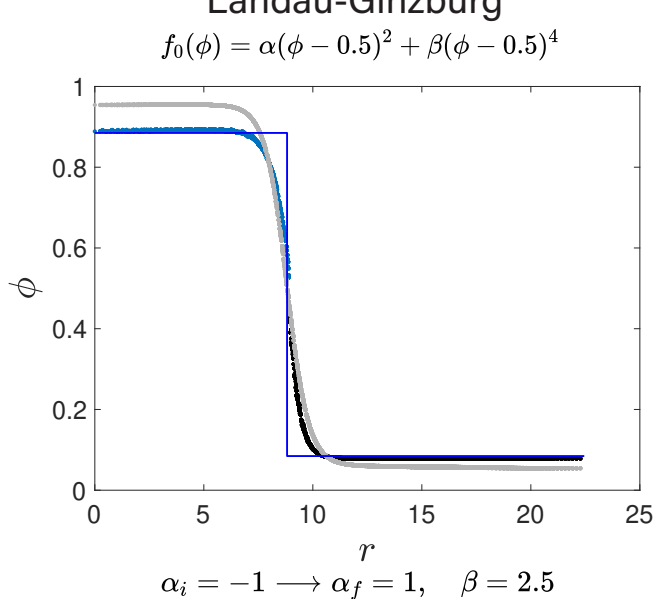

\section{Minimum bulk modulus for stable condensates}

193 In the following, we systematically investigate the in194 dissoluble conditions of elastic condensates. We simulate 195 a condensate with two control parameters $\chi_{f}$ and $G_{B}$ 196 and monitor its dissolution dynamics after $\chi$ is reduced 197 from 3 to $\chi_{f}$. We label the condensate as dissoluble or 198 indissoluble depending on if the system becomes uniform 199 or not after a long waiting time $t=10^{4}$ (Figure $5 \mathrm{~b}, \mathrm{c}$ and 200 Movie S2). As expected, when $G_{B}=0$, the condensate is 201 stable only if $\chi>2$. For $\chi<2$, the condensate becomes 202 indissoluble if the bulk modulus is larger than a critical 203 value $\left(G_{B, c}\right)$. The results are summarized in the phase 204 diagram (Figure 5a).

205 We also succeed in finding the theoretically predicted 206 phase boundary separating the dissoluble and indissolu207 ble phase. We compute the theoretically predicted $\phi_{\text {in }}$ as 208 a function of $G_{B}$ using Eq. (1) with the conservation of 209 total molecular number, and find that $\phi_{\text {in }}$ decreases as ${ }_{210} G_{B}$ decreases (Figure S7). Therefore, a minimum bulk 211 modulus should exist to ensure $\phi_{\text {in }}>\theta \phi_{c}$ so that the elas${ }_{212}$ ticity of the condensate can be maintained. Here, $\theta$ is a ${ }_{213}$ constant presumably close to 1 . We plug $\phi_{\text {in }}=\theta \phi_{c}$ into ${ }_{214}$ Eq. (1) (with $\phi_{\text {out }}$ determined by the conservation of to215 tal molecular number) and note that both $\chi$ and $G_{B}$ ap216 pear linearly in Eq. (1). Therefore, the phase boundary 217 separating dissoluble and indissoluble phase must be lin${ }_{218}$ ear in the $\chi_{f}-G_{B}$ parameter space. Intriguingly, we find 219 that the theoretically computed phase boundary nicely 220 matches the simulated phase diagram (Figure 5a) with ${ }_{221} \theta=1.1$, slightly larger than 1 . Our results are not sen222 sitive to the values of $\phi_{c}$ as we get similar results using 223 different $\phi_{c}$ (Figure S8).

FIG. 4. Simulations using different types of free energy. (a) For the Flory-Huggins free energy, when the control parameter $\chi$ decreases from 3.0 to 1.5, the initial density field (gray dots) cannot be maintained and the final density field is established (blue dots). The blue line is the theoretical prediction based on the equilibrium conditions of elastic condensates. (b) For the Landau-Ginzburg free energy, the control parameter $\alpha$ increases from -1 to 1 , and the equilibrium density field can also be predicted by our theories. In both (a) and (b), a single condensate is simulated, and $G_{S}=20, \phi_{c}=0.5$, $R_{0}=9 . G_{B}=10$ in (a) and $G_{B}=20$ in (b).

${ }_{224}$ To understand why $\theta \gtrsim 1$, we remark that for the elas225 tic condensate to be stable, $\phi_{\text {in }}$ must be larger than $\phi_{c}$. 226 This is the consequence of force balance across the con227 densate boundary. The polymer network is subject to 228 two types of force: the force from the gradient of the 229 osmotic tensor $(\nabla \cdot \boldsymbol{\Pi})$ and the force from the gradient 230 of the elastic stress $(\nabla \cdot \sigma)$. We can further decompose 231 the former force into two parts, one is the from the free ${ }_{232}$ energy $f_{0}(\phi)$, which we call as the osmotic force in the 233 following, and the other is from the $\frac{C}{2}(\nabla \phi)^{2}$ term in the 234 free energy, which we call as the surface tension force 235 in the following. For a liquid condensate, the osmotic ${ }_{236}$ force always balances the surface tension force across the 237 condensate boundary. The crossover regime can be sep${ }_{184} 0$ (Figure $3 \mathrm{~b}$ ) are very close to the predictions with a finite ${ }_{238}$ arated into three parts in which both the surface ten${ }_{185} \gamma$ (Figure S2). To test the generality of our theories, 239 sion force and the osmotic force change their signs (see ${ }_{186}$ we also use the Landau-Ginzburg free energy and find 240 the schematic in Figure 5d and numerical simulations 187 that our theories are equally applicable to both forms of 241 in Figure S9a). For an elastic condensate, the osmotic 188 free energy (Figure 4). Finally, we also test the effects 242 force always points outwards from the condensates since 189 of shear modulus and critical density, and find that our 243 the chemical potential is now a monotonically increasing 190 results are insensitive to the values of $G_{S}$ and $\phi_{c}$ (Figure 244 function of $\phi$. Therefore, in this case, an inward elastic 191 S3-S6), further corroborating our theories.
${ }_{245}$ force must exist to balance the sum of surface tension 
a

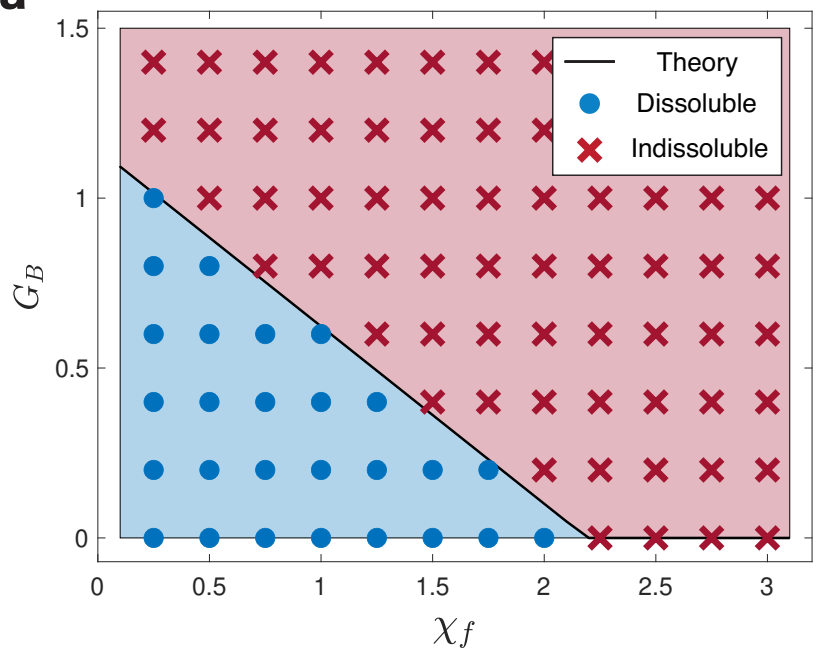

b
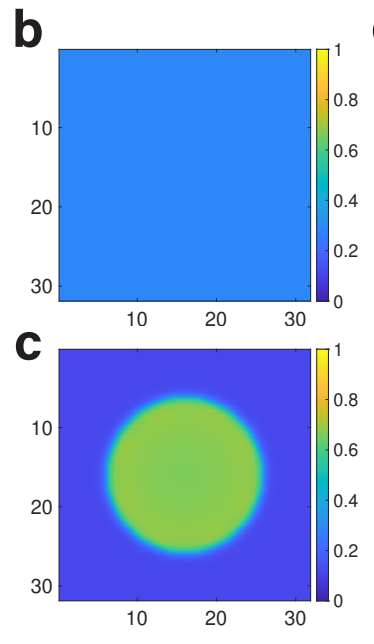

d
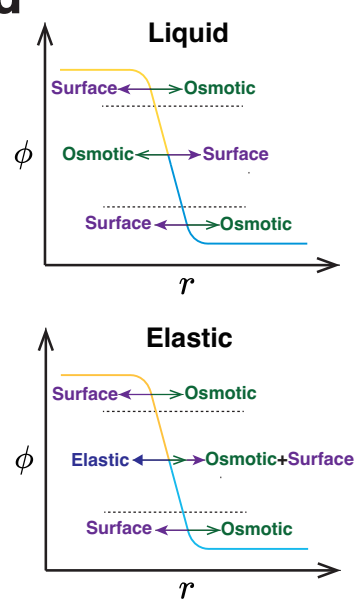

FIG. 5. A critical bulk modulus $G_{B, c}$ above which condensates are indissoluble. (a) Phase diagram of condensate stability with control parameters $\chi_{f}$ and $G_{B}$. The theoretical predicted $G_{B, c}$ is the black line and the simulation results are the blue dots and red crosses. (b) and (c) The density fields $\phi$ for dissolvable and indissoluble cases respectively. In (b), $G_{B}=0.4$ and $\chi_{f}=0.5$ and the final density is uniform. In (c), $G_{B}=1.2$ and $\chi_{f}=1.5$ and the condensate is indissoluble due to elasticity. (d) Schematics for the force balance in the crossover regime of condensates.

246 force and osmotic force in the crossover regime (Figure 275 phase diagram of the dissolution conditions of elastic con${ }_{247} 5 \mathrm{~d}$ and Figure S9b). In conclusion, $\phi_{\text {in }}$ should be larger 276 densates, and obtain a minimum bulk modulus for con${ }_{248}$ than $\phi_{c}$ to ensure a finite elastic force in the crossover 277 densates to be stable upon the condition changes, such 249 regime; therefore, $\theta \gtrsim 1$.

Discussion

251 While the solid-like nature of biomolecular condensates 252 are largely neglected in theoretical modeling, the elastic${ }_{253}$ ity of condensates are crucial to cellular fitness in both ${ }_{254}$ positive and negative ways. The conversion of conden255 sates into a solid-like state could help to preserve bio256 logical structures and suppress detrimental biochemical 286 ${ }_{257}$ reactions $[8,18]$. Furthermore, they help to sequester 258 misfolded proteins $[22,30,31]$. On the other hand, the ${ }^{287}$ 259 dissolution of solid-like condensates may need assistance 260 by energy-consuming enzymes [16, 32-34], therefore, re${ }_{261}$ ducing cellular fitness. Moreover, in conditions where ${ }_{262}$ condensates are supposed to dissolve such as the disso263 lution of stress granules after stress, failure to dissolve 264 may lead to aberrant condensates [8, 18], which further 265 trigger cellular aging and even diseases.

266 Our work provides the first mechanistic understanding 267 on the irreversible nature of aged biomolecular conden-

268 sates. We derive the equilibrium conditions of elastic ${ }_{292}$ Here $f$ is the force field and $\sigma$ is the stress. The above 269 condensates and demonstrate that the bulk stress can 293 equation shows that the elastic energy change can be 270 balance the osmotic pressure difference inside and out- 294 generally expressed as the sum of one bulk term and one ${ }_{271}$ side the condensates, and therefore prevents the disso- 295 surface term.

${ }_{272}$ lution. Our theoretical predictions are nicely confirmed 296 Upon active biological regulation, the attractive inter273 by the numerical simulations using the two-fluid model. 297 action between biomolecules become weaker. In the pres${ }_{274}$ Moreover, we both numerically and theoretically obtain a 298 ence of elasticity, the osmotic pressure difference inside 
299 and outside the condensate can be balanced by the elas- 340 The temporal changing rate of the mixing free energy 300 tic force, which is generated by the deformation field. ${ }_{341} \dot{F}_{\text {mix }}$ is calculated through

301 We consider a condensate with spherical symmetry and 302 the initial displacement field due to the osmotic pressure 303 difference is radial with constant $\nabla \cdot \mathbf{u}_{\mathbf{p}}$. This gener304 ates a dilution of density in bulk of condensate such that ${ }_{305} \nabla \cdot \mathbf{u}_{\mathbf{p}}=\log \left(\phi_{1} / \phi_{\text {in }}\right)$ where $\phi_{1}\left(\phi_{\text {in }}\right)$ is the density before 306 (after) the biological regulation. Note that the stress 307 field inside the condensate is diagonal and also constant $308 \sigma=\sigma_{B} \mathbf{I}$.

309 Now we consider a small change in the volume 310 of the condensate without change in the number of 311 biomolecules. This leads to a change in the density: ${ }_{312} \delta \phi / \phi=-\delta V / V$. The resulting change in the displace313 ment field therefore satisfies $\nabla \cdot \delta \mathbf{u}_{\mathbf{p}}=-\delta \phi / \phi=\delta V / V$. ${ }_{314}$ Using the spherical symmetry, we obtain $\delta \mathbf{u}_{\mathbf{p}}(r)=$ ${ }_{315} r \delta V /(V \cdot d) \mathbf{e}_{\mathbf{r}}$ where $d$ is the spatial dimension and $\mathbf{e}_{\mathbf{r}}$ 316 is the unit radial vector. Therefore, near the surface, 317 the change in the bulk displacement field $\delta \mathbf{u}_{\mathbf{p}}(r \rightarrow R)=$ ${ }_{318} R \delta V /(V \cdot d) \mathbf{e}_{\mathbf{r}}$. Interestingly, $\delta \mathbf{u}_{\mathbf{p}}(r \rightarrow R)$ is just the dis319 placement change needed to increase the volume by $\delta V$. 320 This means that the displacement change on the surface 321 is zero, and only the bulk term contributes to the elas322 tic energy change. Therefore, the change in the elastic 323 energy becomes

$$
\delta F_{e l}=\sigma_{B} \delta V
$$

324 Since the free energy changes due to the osmotic pressure 325 and surface tension are the same as the usual liquid con${ }_{326}$ densate, the equilibrium condition due to volume change 327 becomes

$$
\Pi_{\text {in }}-\sigma_{B}=\Pi_{\text {out }}+\frac{(d-1) \gamma}{R} .
$$

329 Numerical simulations of viscoelastic phase separation 330 are based on the two-fluid model $[24,25]$, which consid331 ers the dynamics of polymer velocity $\mathbf{v}_{\mathbf{p}}$, solvent velocity ${ }_{332} \mathbf{v}_{\mathbf{s}}$ and the average velocity $\mathbf{v}=\phi \mathbf{v}_{\mathbf{p}}+(1-\phi) \mathbf{v}_{\mathbf{s}}$. This 333 model is derived by minimizing the Rayleighian $R$ of the 334 solution $[24,25,35]$, the sum of the energy dissipation 335 function $\Phi$ and the temporal changing rate of the free ${ }_{336}$ energy $\dot{F}$. The energy dissipation function $\Phi$ consists ${ }_{337}$ of two parts, which are respectively the friction between 338 polymer and solvent $\Phi_{1}$, and the overall viscous dissipa339 tion of the solution $\Phi_{2}$

$$
\begin{aligned}
& \Phi_{1}=\int \mathrm{d} \mathbf{r} \frac{\zeta}{2}\left(\mathbf{v}_{\mathbf{p}}-\mathbf{v}_{\mathbf{s}}\right)^{2}=\int \mathrm{d} \mathbf{r} \frac{1}{2} \zeta \frac{\left(\mathbf{v}_{\mathbf{p}}-\mathbf{v}\right)^{2}}{(1-\phi)^{2}} \\
& \Phi_{2}=\int \mathrm{d} \mathbf{r} \frac{\eta}{4}\left(\nabla \mathbf{v}+(\nabla \mathbf{v})^{T}\right):\left(\nabla \mathbf{v}+(\nabla \mathbf{v})^{T}\right) .
\end{aligned}
$$

$$
\begin{aligned}
\dot{F}_{\text {mix }} & =\int \mathrm{d} \mathbf{r} \dot{\phi} f^{\prime}(\phi)=\int \mathrm{d} \mathbf{r}\left[-\nabla \cdot\left(\phi \mathbf{v}_{\mathbf{p}}\right)\right] f^{\prime}(\phi) \\
& \left.=\int \mathrm{d} \mathbf{r}\left[\phi \nabla f^{\prime}(\phi)\right)\right] \cdot \mathbf{v}_{\mathbf{p}}=\int \mathrm{d} \mathbf{r}(\nabla \cdot \mathbf{\Pi}) \cdot \mathbf{v}_{\mathbf{p}},
\end{aligned}
$$

342 involving the continuous equation of the density: $\dot{\phi}=$ ${ }^{343}-\nabla \cdot\left(\phi \mathbf{v}_{\mathbf{p}}\right)$. Here $f(\phi)=f_{0}(\phi)+\frac{C}{2}(\nabla \phi)^{2}$ and $C$ is a 344 constant. The elastic energy comes from the polymer, so 345 its temporal changing rate is

$$
\dot{F}_{\mathrm{el}}=\int \mathrm{d} \mathbf{r} \sigma_{i j} \partial_{j} v_{p_{i}}=\int \mathrm{d} \mathbf{r}(-\nabla \cdot \sigma) \cdot \mathbf{v}_{\mathbf{p}}
$$

${ }_{346}$ Combined with the constrain from the incompressible 347 condition

$$
\nabla \cdot \mathbf{v}=0
$$

${ }_{348}$ and all components mentioned above, the Rayleighian of 349 the solution is therefore:

$$
\begin{aligned}
R= & \int \mathrm{d} \mathbf{r}\left[-p(\nabla \cdot \mathbf{v})+\frac{\zeta}{2} \frac{\left(\mathbf{v}_{\mathbf{p}}-\mathbf{v}\right)^{2}}{(1-\phi)^{2}}\right. \\
& +\frac{\eta}{4}\left(\nabla \mathbf{v}+(\nabla \mathbf{v})^{T}\right):\left(\nabla \mathbf{v}+(\nabla \mathbf{v})^{T}\right) \\
& \left.+(\nabla \cdot \boldsymbol{\Pi}) \cdot \mathbf{v}_{\mathbf{p}}-(\nabla \cdot \sigma) \cdot \mathbf{v}_{\mathbf{p}}\right]
\end{aligned}
$$

${ }_{350}$ By setting the functional derivative of $R$ with $\mathbf{v}_{\mathbf{p}}$ and $\mathbf{v}$ 351 to be 0 , we obtain the following equations:

$$
\begin{aligned}
& \frac{\zeta}{(1-\phi)^{2}}\left(\mathbf{v}_{\mathbf{p}}-\mathbf{v}\right)+\nabla \cdot \boldsymbol{\Pi}-\nabla \cdot \sigma=0 \\
& \nabla p-\frac{\zeta}{(1-\phi)^{2}}\left(\mathbf{v}_{\mathbf{p}}-\mathbf{v}\right)-\eta \nabla^{2} \mathbf{v}=0
\end{aligned}
$$

352 Finally, we rewrite the above equations and obtain

$$
\begin{aligned}
& \frac{\partial \phi}{\partial t}=-\nabla \cdot\left(\phi \mathbf{v}_{\mathbf{p}}\right), \\
& \mathbf{v}_{\mathbf{p}}-\mathbf{v}=-\frac{(1-\phi)^{2}}{\zeta}(\nabla \cdot \boldsymbol{\Pi}-\nabla \cdot \sigma), \\
& -\nabla \cdot \boldsymbol{\Pi}+\nabla \cdot \sigma-\nabla p+\eta \nabla^{2} \mathbf{v}=0
\end{aligned}
$$

${ }_{353}$ Clearly, Eq. (5) is obtained from Eq. (19) and Eq. (20). 354 Combined with the incompressible condition $\nabla \cdot \mathbf{v}=0$ 355 and Eq. (21), the average velocity $\mathbf{v}$ is calculated as

$$
\mathbf{v}(\mathbf{r})=\int \mathrm{d} \mathbf{r}^{\prime} \mathbf{T}\left(\mathbf{r}-\mathbf{r}^{\prime}\right) \cdot\left(-\nabla \cdot \boldsymbol{\Pi}\left(\mathbf{r}^{\prime}\right)+\nabla \cdot \sigma\left(\mathbf{r}^{\prime}\right)\right),
$$

356 while $\mathbf{T}(\mathbf{k})=\frac{1}{\eta|\mathbf{k}|^{2}}\left(\mathbf{I}-\frac{\mathbf{k k}}{|\mathbf{k}|^{2}}\right)$ is the Oseen tensor in the ${ }_{357}$ Fourier space. We can then obtain the polymer veloc358 ity $\mathbf{v}_{\mathbf{p}}$ with equation (20). Therefore, the density $\phi$ in 359 simulation can be updated by calculating $\mathbf{v}_{\mathbf{p}}$ when the 360 osmotic pressure $\boldsymbol{\Pi}$ and the stress $\sigma$ are known. 
361 The two components in stress tensor $\sigma$, which are shear 395 362 stress tensor $\sigma_{S}$ and bulk stress scalar $\sigma_{B}$, respectively 396 363 obey the following Maxwell-type equations [25]:

$$
\begin{aligned}
\frac{\partial \sigma_{S}}{\partial t}= & -\left(\mathbf{v}_{\mathbf{p}} \cdot \nabla\right) \sigma_{S}+\sigma_{S} \cdot \nabla \mathbf{v}_{\mathbf{p}}+\left(\nabla \mathbf{v}_{\mathbf{p}}\right)^{T} \cdot \sigma_{S} \\
& -\frac{1}{\tau_{S}(\phi)} \sigma_{S}+G_{S}(\phi)\left(\nabla \mathbf{v}_{\mathbf{p}}+\left(\nabla \mathbf{v}_{\mathbf{p}}\right)^{T}\right) \\
\frac{\partial \sigma_{B}}{\partial t}= & -\left(\mathbf{v}_{\mathbf{p}} \cdot \nabla\right) \sigma_{B}-\frac{1}{\tau_{B}(\phi)} \sigma_{B}+G_{B}(\phi) \nabla \cdot \mathbf{v}_{\mathbf{p}}
\end{aligned}
$$

364 In our simulations, we first do not include elastic stress ${ }^{407}$ 365 to form condensates by taking $G_{S}=G_{B}=0$. We then 366 introduce the elastic stress by taking

$$
\begin{aligned}
G_{S}(\phi) & =G_{S} \phi^{2}, \\
\tau_{S}(\phi) & =\frac{1}{\phi_{c}-\phi} \Theta\left(\phi_{c}-\phi\right), \\
G_{B}(\phi) & =G_{B} \Theta\left(\phi-\phi_{c}\right), \\
\tau_{B}(\phi) & =\frac{1}{\phi_{c}-\phi} \Theta\left(\phi_{c}-\phi\right) .
\end{aligned}
$$

${ }_{367}$ Here $G_{B}$ and $G_{S}$ are constants. We assume a critical ${ }_{420}^{410}$ 368 density $\phi_{c}$ above which the polymer network is percolated 369 and becomes fully elastic with a finite bulk modulus and 422 370 a diverging relaxation time.

\section{Details of numerical simulations}

372 We perform numerical simulations in a 2D grid by solv373 ing the two-fluid model using the explicit Euler method 374 with the periodic boundary condition on MATLAB. Sim375 ulations for a single condensate are in a $127 \times 127$ 376 grid, and simulations for multiple condensates are in a $377255 \times 255$ grid. The grid size is $\Delta L=0.25$ by set378 ting the unit length equal to the lattice size when de379 riving the mixing free energy. The time interval for the 380 simulation is $\Delta t=0.001$. In our simulations, we take ${ }_{381} k_{B} T=C=\xi=\eta=1$ for simplicity. The elastic 382 stress is introduced at $t=10^{3}$ for a single condensate з8з and $t=4 \times 10^{3}$ for multiple condensates. The control 384 parameter $\chi$ is changed 50 time units after adding the 385 elasticity. Eq. (22) is solved with fast Fourier transfor386 mation and other equations are calculated in real space. 387 For the simulation of multiple condensates, we initially 388 add a Gaussian noise with variance 0.001 to the uniform 389 density field. In Figure 5a, simulations are initiated with 390 a single condensate with $R_{0}=9$. The condensate is con391 sidered dissoluble if the variance of the $\phi$ field at $t=10^{4}$ 392 is less than 0.01 .

[1] C. P. Brangwynne, C. R. Eckmann, D. S. Courson, 457 394 A. Rybarska, C. Hoege, J. Gharakhani, F. Jülicher, and ${ }_{458}$
A. A. Hyman, Germline p granules are liquid droplets that localize by controlled dissolution/condensation, Science 324, 1729 (2009).

[2] C. P. Brangwynne, P. Tompa, and R. V. Pappu, Polymer physics of intracellular phase transitions, Nature Physics 11, 899 (2015).

[3] A. Patel, H. O. Lee, L. Jawerth, S. Maharana, M. Jahnel, M. Y. Hein, S. Stoynov, J. Mahamid, S. Saha, T. M. Franzmann, et al., A liquid-to-solid phase transition of the als protein fus accelerated by disease mutation, Cell 162, 1066 (2015).

[4] Y. Lin, D. S. Protter, M. K. Rosen, and R. Parker, Formation and maturation of phase-separated liquid droplets by rna-binding proteins, Molecular cell 60, 208 (2015).

[5] S. F. Banani, H. O. Lee, A. A. Hyman, and M. K. Rosen, Biomolecular condensates: organizers of cellular biochemistry, Nature reviews Molecular cell biology 18, 285 (2017).

[6] J. B. Woodruff, B. F. Gomes, P. O. Widlund, J. Mahamid, A. Honigmann, and A. A. Hyman, The centrosome is a selective condensate that nucleates microtubules by concentrating tubulin, Cell 169, 1066 (2017).

[7] Y. Shin, J. Berry, N. Pannucci, M. P. Haataja, J. E. Toettcher, and C. P. Brangwynne, Spatiotemporal control of intracellular phase transitions using light-activated optodroplets, Cell 168, 159 (2017).

[8] T. M. Franzmann, M. Jahnel, A. Pozniakovsky, J. Mahamid, A. S. Holehouse, E. Nüske, D. Richter, W. Baumeister, S. W. Grill, R. V. Pappu, A. A. Hyman, and S. Alberti, Phase separation of a yeast prion protein promotes cellular fitness, Science 359 (2018).

[9] J. Wang, J.-M. Choi, A. S. Holehouse, H. O. Lee, X. Zhang, M. Jahnel, S. Maharana, R. Lemaitre, A. Pozniakovsky, D. Drechsel, et al., A molecular grammar governing the driving forces for phase separation of prion-like rna binding proteins, Cell 174, 688 (2018).

[10] S. Alberti, A. Gladfelter, and T. Mittag, Considerations and challenges in studying liquid-liquid phase separation and biomolecular condensates, Cell 176, 419 (2019).

[11] A. S. Lyon, W. B. Peeples, and M. K. Rosen, A framework for understanding the functions of biomolecular condensates across scales, Nature Reviews Molecular Cell Biology 22, 215 (2021).

[12] L. M. Jawerth, M. Ijavi, M. Ruer, S. Saha, M. Jahnel, A. A. Hyman, F. Jülicher, and E. Fischer-Friedrich, Salt-dependent rheology and surface tension of protein condensates using optical traps, Phys. Rev. Lett. 121, 258101 (2018).

[13] L. Jawerth, E. Fischer-Friedrich, S. Saha, J. Wang, T. Franzmann, X. Zhang, J. Sachweh, M. Ruer, M. Ijavi, S. Saha, J. Mahamid, A. A. Hyman, and F. Jülicher, Protein condensates as aging maxwell fluids, Science $\mathbf{3 7 0}$, 1317 (2020).

[14] A. Molliex, J. Temirov, J. Lee, M. Coughlin, A. P. Kanagaraj, H. J. Kim, T. Mittag, and J. P. Taylor, Phase separation by low complexity domains promotes stress granule assembly and drives pathological fibrillization, Cell 163, 123 (2015).

54 [15] S. Alberti and A. A. Hyman, Are aberrant phase transitions a driver of cellular aging?, BioEssays 38, 959 (2016).

[16] J. A. Riback, C. D. Katanski, J. L. Kear-Scott, E. V. Pilipenko, A. E. Rojek, T. R. Sosnick, and D. A. Drummond, Stress-triggered phase separation is an adaptive, 
evolutionarily tuned response, Cell 168, 1028 (2017).

493

T. S. Harmon, A. S. Holehouse, M. K. Rosen, and R. V.
Pappu, Intrinsically disordered linkers determine the interplay between phase separation and gelation in multi- 496 valent proteins, eLife 6, e30294 (2017).

18] C. Iserman, C. Desroches Altamirano, C. Jegers, 498 U. Friedrich, T. Zarin, A. W. Fritsch, M. Mittasch, 499 A. Domingues, L. Hersemann, M. Jahnel, D. Richter, U.- 500 P. Guenther, M. W. Hentze, A. M. Moses, A. A. Hyman, 501 G. Kramer, M. Kreysing, T. M. Franzmann, and S. Al- 502 berti, Condensation of ded1p promotes a translational ${ }_{503}$ switch from housekeeping to stress protein production, 504 Cell 181, 818 (2020).

19] A. K. Rai, J.-X. Chen, M. Selbach, and L. Pelkmans, 506 Kinase-controlled phase transition of membraneless or- 507 ganelles in mitosis, Nature 559, 211 (2018).

20] Y. Shin and C. P. Brangwynne, Liquid phase condensation in cell physiology and disease, Science 357 (2017). $\quad 510$

21] S. Alberti and D. Dormann, Liquid-liquid phase sepa- 511 ration in disease, Annual Review of Genetics 53, 171512 (2019), pMID: 31430179.

22] S. Alberti and A. A. Hyman, Biomolecular condensates 514 at the nexus of cellular stress, protein aggregation disease 515 and ageing, Nature Reviews Molecular Cell Biology 22, 516 196 (2021).

23] J. Berry, C. P. Brangwynne, and M. Haataja, Physical 518 principles of intracellular organization via active and pas- 519 sive phase transitions, Reports on Progress in Physics 81, 520 046601 (2018).

24] H. Tanaka, Viscoelastic phase separation, Journal of ${ }_{522}$ Physics: Condensed Matter 12, R207 (2000).

H. Tanaka and T. Araki, Viscoelastic phase separation ${ }_{524}$ in soft matter: Numerical-simulation study on its phys- 525 ical mechanism, Chemical Engineering Science 61, 2108526
(2006).

[26] P. C. Hohenberg and B. I. Halperin, Theory of dynamic critical phenomena, Rev. Mod. Phys. 49, 435 (1977).

[27] X. Wei, J. Zhou, Y. Wang, and F. Meng, Modeling elastically mediated liquid-liquid phase separation, Phys. Rev. Lett. 125, 268001 (2020).

[28] Y. Zhang, D. S. W. Lee, Y. Meir, C. P. Brangwynne, and N. S. Wingreen, Mechanical frustration of phase separation in the cell nucleus by chromatin, Phys. Rev. Lett. 126, 258102 (2021).

[29] P. Ronceray, S. Mao, A. Košmrlj, and M. P. Haataja, Liquid demixing in elastic networks: cavitation, permeation, or size selection? (2021), arXiv:2102.02787 [condmat.soft].

[30] D. Kaganovich, R. Kopito, and J. Frydman, Misfolded proteins partition between two distinct quality control compartments, Nature 454, 1088 (2008).

[31] S. Escusa-Toret, W. I. Vonk, and J. Frydman, Spatial sequestration of misfolded proteins by a dynamic chaperone pathway enhances cellular fitness during stress, Nature cell biology 15, 1231 (2013).

[32] S. Jain, J. R. Wheeler, R. W. Walters, A. Agrawal, A. Barsic, and R. Parker, Atpase-modulated stress granules contain a diverse proteome and substructure, Cell 164, 487 (2016).

[33] M. Hondele, R. Sachdev, S. Heinrich, J. Wang, P. Vallotton, B. M. Fontoura, and K. Weis, Dead-box atpases are global regulators of phase-separated organelles, Nature 573, 144 (2019).

[34] D. Tauber, G. Tauber, A. Khong, B. Van Treeck, J. Pelletier, and R. Parker, Modulation of rna condensation by the dead-box protein eif4a, Cell 180, 411 (2020).

[35] M. Doi, Soft matter physics (Oxford University Press, 2013). 